\title{
Molecular pathways governing development of vascular endothelial cells from ES/iPS cells
}

\begin{abstract}
Assembly of complex vascular networks occurs in numerous biological systems through morphogenetic processes such as vasculogenesis, angiogenesis and vascular remodeling. Pluripotent stem cells such as embryonic stem (ES) and induced pluripotent stem (iPS) cells can differentiate into any cell type, including endothelial cells (ECs), and have been extensively used as in vitro models to analyze molecular mechanisms underlying EC generation and differentiation. The emergence of these promising new approaches suggests that ECs could be used in clinical therapy. Much evidence suggests that ES/iPS cell differentiation into ECs in vitro mimics the in vivo vascular morphogenic process. Through sequential steps of maturation, ECs derived from ES/iPS cells can be further differentiated into arterial, venous, capillary and lymphatic ECs, as well as smooth muscle cells. Here, we review EC development from ES/iPS cells with special attention to molecular pathways functioning in EC specification.
\end{abstract}

Keyword: Endothelial cells; Differentiation; Molecular pathways; Embryonic stem cells; Induced pluripotent stem cells 